\title{
Estimating the terms of turbulent kinetic energy transport equation in an unsteady flow on the basis of SIV measurements
}

\author{
Nikolay Mikheev ${ }^{1, *}$ and Ilya Saushin ${ }^{1}$ \\ ${ }^{1}$ Kazan Scientific Center of RAS, Kazan, 420111, Russia \\ ${ }^{2}$ Kazan National Research Technical University named after A.N. Tupolev, Kazan, 420111, Russia
}

\begin{abstract}
Turbulence production and dissipation profiles averaged over a period of forced flow pulsations have been obtained experimentally across the boundary layer, developing on a channel wall under forced periodic freestream unsteadiness. The experiments have been conducted in a wide range of dimensionless numbers $\beta=0.03-0.28$ and Strouhal numbers $\mathrm{St}=2.99-64.2$ that characterize relative amplitude and frequency of flow pulsations. The parameters have been estimated based on the instantaneous velocity fields, measured by $2 \mathrm{D}$ optical method of Smoke Image Velocimetry (SIV). Turbulence dissipation and production profiles plotted in wall coordinates have been compared to the corresponding steady flow profiles. In the considered ranges of dimensionless numbers, the insensitivity of turbulent dissipation profiles to periodic flow unsteadiness has been established, and conclusion on similar insensitivity of turbulence production profiles drawn on the basis of point measurements and DNS has been confirmed.
\end{abstract}

\section{Introduction}

A transport equation of turbulent kinetic energy is a basic equation describing turbulence production, transport and dissipation. In respect to the boundary layer, it is important to have the information on energy balance across the boundary layer. It enables a further insight into complex processes and phenomena, a study laminar-turbulent transition and updating of turbulence models intended for RANS and LES methods.

Turbulence production in the boundary layer is written through a product of the second moments of velocity fluctuations and the spatial gradient of velocity

$$
\left\langle P_{i j}\right\rangle=\left\langle u_{i}^{\prime} u_{j}^{\prime}\right\rangle \frac{\partial\left\langle U_{i}\right\rangle}{\partial x_{j}} .
$$

This term was estimated in [1-5] for the turbulent boundary layer under forced freestream pulsations of the flow around a plate and in a tube in a wide range of

*Corresponding author: n.miheev@mail.ru 
dimensionless numbers. These estimates were obtained using point measurements and included, among others, the data on dynamics of turbulence production profiles over the phase of forced pulsations. Authors $[6,7]$ analyzed the influence of forced unsteadiness on the production term theoretically on the basis of turbulent fluctuation measurements.

To calculate turbulence dissipation, pairwise correlations of spatial gradients of pulsatory components of all three velocity vector components should be estimated:

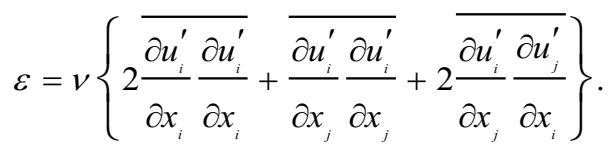

Obviously, point measurements cannot yield a direct estimation of $\varepsilon$. Authors [5] analyzed the behavior of dissipation integrated over the boundary layer thickness depending on the phase of forced pulsations. The dissipation was estimated by the difference between the turbulent kinetic energy and its production.

Authors [8-10] noted that dissipation cannot be estimated by an optical technique of Particle Image Velocimetry (PIV) with sufficient accuracy as yet due to limited spatial resolution of the method. The potential of 3D particle tracking (LPT) in particle trajectory reconstruction in high seeding density flows was shown in [11] using Shake-The-Box technique. This will obviously allow the direct estimation of the strain rate tensor components and hence dissipation. Yet the development of experimental methods for estimation of dissipation in the boundary layer remains an open problem.

The paper deals with estimation of production and dissipation profiles averaged over the period of forced pulsations in pulsating turbulent boundary layer using the instantaneous velocity fields measured by an optical method of Smoke Image Velocimetry (SIV) [12].

\section{Experimental technique and procedure}

An experimental setup shown in fig. 1 was used to study the boundary layer structure in the pulsating flow. Test section 1 of the experimental setup was a $75 \times 150 \mathrm{~mm}^{2}$ one meter long rectuangular channel with a $6: 1$ contraction ratio smooth inlet 10 . Turbulence generating grid 9 with square cells was mounted downstream of the smooth inlet. The grid was made of 1.2-mm thick wire; the distance between the wire axes was $5 \mathrm{~mm}$. A $50-\mathrm{mm}$ long strip of abrasive 12 (ISO 6344-2) was glued onto the channel perimeter. This provided fully developed turbulent boundary layer in the measurement area during the experiments. Channel walls were made of transparent materials (glass and polycarbonate). Stable air flow rate downstream of the test section was provided by a regulating gate 11 and a $1.3 \mathrm{~m}^{3}$ receiver tank 2 . The flow rate was measured by an ultrasonic flowmeter IRVIS RS4-Ultra 3 mounted downstream of the receiver tank. The relative error in flow rate did not exceed $1 \%$. The average flow rate, frequency and amplitude of forced velocity pulsations were adjusted by a static and rotating flap in pulsator 13 .

To visualize the flow pattern, the air-aerosol mixture (MT-Gravity fluid with medium fog density and average particle size of $0.1 \ldots 5 \mu \mathrm{m}$; Safex aerosol generator 5) was supplied from the preparation chamber 4 to the channel inlet. The measurement area 6 was illuminated by a continuous diode-pumped solid-state laser KLM-532/5000-h 7. The flow pattern in the channel symmetry plane at the distance of $L=0.7 \mathrm{~m}$ from the turbulence generating grid was recorded by a monochrome high-speed camera Fastec HiSpec 8 with the frame resolution of $665 \times 110$ pixel (scaling factor of $0.0625 \mathrm{~mm} / \mathrm{pixel}$ ), frame rate $f=$ $70831 / \mathrm{s}$, and recording time of $3 \mathrm{~s}$.

Flow velocity fields were measured by the optical method of Smoke Image Velocimetry (SIV) [12], in which velocity fields are obtained from the analysis of turbulent structure 
displacements visualized by smoke. Period-averaged frame resolution in $\mathrm{y}^{+}$-coordinates was 1 pixel $=0.8 \mathrm{y}^{+}$, interrogation window size was $16 \times 16$ pixel, period-averaged ratio of the resolved scale to Kolmogorov length scale was 1.6.

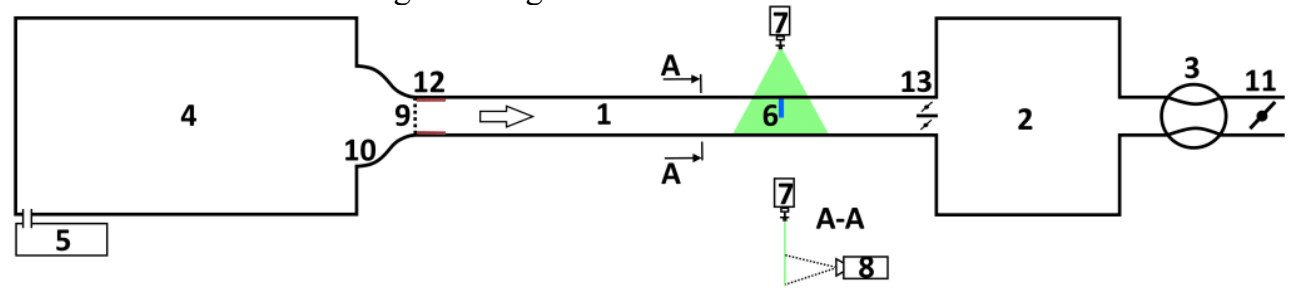

Fig. 1. Experimental setup

\section{Results and discussion}

Turbulence production and dissipation were estimated by turbulent velocity fluctuations. The latter were obtained from the following relation [13] (for the case of forced periodic freestream pulsations):

$$
u_{i}^{\prime}(y, t)=U(y, t)-\bar{U}(y)-\tilde{U}(y, t),
$$

where $U$ is the instantaneous velocity, $\bar{U}$ is the average instantaneous velocity, $\tilde{U}$ is the fundamental frequency of $U$ oscillogram corresponding to the frequency of forced unsteadiness. Second-order central difference scheme was used to approximate the derivatives. Dissipation was estimated with the assumption of turbulence isotropy in transversal direction.

Fig. 2 shows the period-averaged profiles of turbulence production and generation in wall coordinates. The profiles are normalized by $v / \overline{\mathrm{u}}_{\tau}^{4}$ and illustrate five regimes of pulsating flow and steady flow $(\mathrm{St}=0)$ considered in the research. Similar profiles obtained by DNS $[14,15]$ are plotted with lines. Experimental data for the steady flow case were obtained for two sizes of the measurement area along $y$-coordinate: 6 and 16 pixel (approximately $5 \ldots 13$ in wall coordinates). As one can see from the figure, the peak of turbulence production curve is significantly "smeared" due to spatial averaging. This indicates that the measurement area should be reduced in the wall-normal direction down to the order of Kolmogorov length scale.

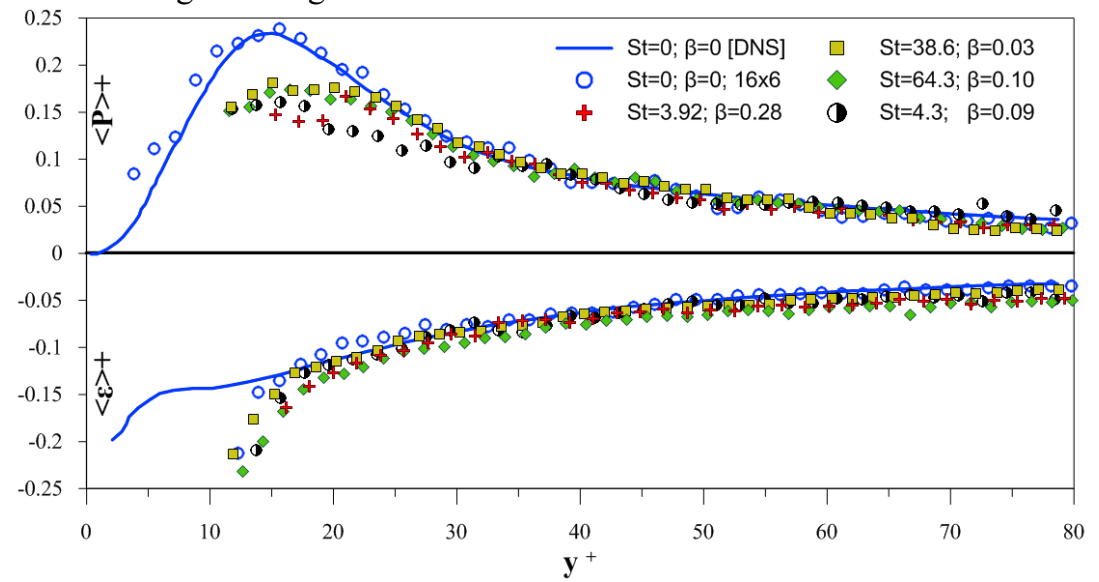

Fig. 2. Period-averaged profiles of turbulence production and dissipation in the boundary layer of steady and pulsating flow; DNS: $<\mathrm{P}>+[14],<\varepsilon>+[15]$ 
Fig.2 shows that despite the wide range of dimensionless Strouhal number, St, and relative amplitude of velocity, $\beta$, on the channel axis considered in the research, almost identical profiles of turbulence production and dissipation were obtained. As for the turbulence generation profile, this result agrees with conclusions drawn in [1-3, 16] on the insensitivity of $\langle\mathrm{P}\rangle^{+}$profile to the forced periodic unsteadiness revealed by point measurements and DNS. Dissipation profiles in the turbulent boundary layer in conditions of forced flow unsteadiness have been obtained in the present paper for the first time ever. These profiles appeared to be insensitive to the periodic unsteadiness.

\section{Conclusions}

Space and time scales of velocity vector fields resolved by SIV technique are sufficient for the estimation of velocity fluctuation derivatives with respect to time and space. Turbulent energy production and dissipation profiles in the pulsating flow in the channel have been estimated from SIV measurements. The insensitivity of turbulent energy production and dissipation profiles to periodic flow unsteadiness has been experimentally established in the considered range of dimensionless numbers.

This work was supported by the Russian Science Foundation (Project no.16-19-10336).

\section{References}

1. G.J. Brereton, W.C. Reynolds, R. Jayaraman, J. Fluid Mech. 221, 131 (1990)

2. G.J. Brereton, W.C. Reynolds, Phys. Fluids A 3, 178 (1991)

3. R. Akhavan, R.D. Kamm, A.H. Shapiro, J. Fluid Mech. 225, 395 (1991)

4. M.M. Grigoryev,V.V. Kuzmin, A.V. Fafurin, J. Eng Phys Thermophys 59, 725

5. M.Hino, M.Kashiwayanagi, A.Nakayama, T. Hara, J. Fluid Mech. 131, 363 (1983)

6. F.S. Tardu, G. Binder, R.F. Blackwelder, J. Fluid Mech. 267, 109 (1994)

7. G. Binder, S. Tardu, P. Vezin, Proc. R. Soc. Lond. 451, 121 (1995)

8. J. Charonko, K. Prestridge, 18th International Symposium on the Application of Laser and Imaging Techniques to Fluid Mechanics, ISBN 978-989-98777-8-8 (2016)

9. J.M. Foucaut, C. Cuvier, M. Stanislas, W.K. George, Progress in wall turbulence 2. Ercoftac series 23, 429 (2016)

10. J.F.G. Schneiders, F. Scarano, Exp Fluids 57, 139 (2016)

11. A. Schröder, D. Schanz, R. Geisler, S. Gesemann, 18th International Symposium on the Application of Laser and Imaging Techniques to Fluid Mechanics, ISBN 978-98998777-8-8 (2016)

12. N.I. Mikheev, N.S. Dushin, Instrum. Exp. Tech. 59, 880 (2016)

13. A.K.M.F. Hussain, W.C. Reynolds, J. Fluid Mech. 41, 241 (1970)

14. N.N. Mansour, J.Kim, P. Moin, J. Fluid Mech. 194, 15 (1988)

15. P. Schlatter, R. Örlü, J. Fluid Mech. 659, 116 (2016)

16. P.K. Papadopoulos, A.P. Vouros, Int. J. Heat Fluid Flow 58, 54, (2016) 